\title{
Electric Field Modelling in Gas-Insulated Substation for Analysis of Conditions for Partial Discharge Phenomena
}

\author{
B. FlorkowskA ${ }^{a, *}$, P. ZYdroñ ${ }^{a}$ AND A. JACKOWICZ-KORCZYŃSKI ${ }^{b}$ \\ ${ }^{a}$ AGH - University of Science and Technology, Department of Electrical and Power Engineering, \\ al. Mickiewicza 30, 30-059 Kraków, Poland \\ ${ }^{b}$ Stator Service Polska, al. Roździeńskiego 188, 40-203 Katowice, Poland \\ (Received January 3, 2015; in final form August 6, 2015)
}

\begin{abstract}
The paper refers to diagnostics problems connected with the presence of partial discharges in gas-insulated substation. The basic stimulus for partial discharges generation is local, high value electric field, greater than the inception one. The numerical simulations of the electric field distribution in the part of the gas insulating system and in the vicinity of the metallic protrusion located on high voltage conductor are presented. Such defects are often present in $\mathrm{SF}_{6}$ insulated constructions and are the cause of a local increase of the electric field. Theoretical analysis of physical mechanism of partial discharges initiation and development in the case of micro-needle type defects is described.
\end{abstract}

DOI: 10.12693 /APhysPolA.128.319

PACS: $51.50 .+\mathrm{v}, 52.80 .-\mathrm{s}, 84.70 .+\mathrm{p}, 41.20 .-\mathrm{q}$

\section{Introduction}

Gas-insulated substations (GISs) with sulphur hexafluoride $\left(\mathrm{SF}_{6}\right)$ have been used for many years because of excellent insulating and arc extinguishing properties of $\mathrm{SF}_{6}$. Gas-insulated constructions are used in medium and high voltage substations. On the basis of many years experience all over the world it has been concluded that reliability of substations, limited by insulating system dielectric performance can be treated satisfactory for the arrangements up to nominal voltage $170 \mathrm{kV}$. Currently, the main development and application of gas insulated substations concerns the high- and extra high voltages [1-3]. Deterioration of gas due to partial discharge (PD) phenomena is the main problem of insulating systems with $\mathrm{SF}_{6}$. The sulphur hexafluoride practically does not degrade in-service at the working electric field stress if there are no partial discharges. When $\mathrm{SF}_{6}$ gas is exposed to increased electric field and partial discharges are present, the gas decomposes into various products, depending on the discharge energy, water content and purity of gas. The insulation performance of $\mathrm{SF}_{6}$ degrades under inhomogeneous electric field increased mainly under the influence of conducting defects on the conductors or on the surface of insulators. The danger of partial discharges is real and has essential meaning for reliable operation of modern gas insulated substations. Elimination of partial discharges from GIS constructions, their preventive detection and development of new diagnostics methods are demanded [4]. The GIS monitoring allowing detection of the PD signals inside the GIS construction using electrical method, conventional and special $U H F / V H F$ have

\footnotetext{
* corresponding author; e-mail: beflor@agh.edu.pl
}

evolved [5-13]. The physicochemical methods of GIS monitoring based on, e.g. gas spectroscopy method [14] or application of carbon nanotube gas sensor [15] were also reported.

The gas-insulated substation has a complicated design and contains elements which differ substantially in terms of the electric field distribution. The GIS design optimization refers to the selection of the permissible value of the electric field in the insulating system due to the dielectric strength of $\mathrm{SF}_{6}$, but also thermal and mechanical properties of the GIS construction [16]. This condition may not be sufficient in the case of a local increase of the electric field in a gas insulating system, at which partial discharges are initiated. Being the basic task at preliminary stage of new substation project, the analysis of electric field distribution in substations modules is the basis for the recognition and description of conditions of PD appearance in gas-insulated substations. Deformation of electric field and its local increase during normal operation are usually effects of the presence of metallic protrusion or micro-needles in insulating system of the substation $[17,18]$.

The above problem is presented in the article on the example of a spherical connector, one of the construction elements of GIS. The electric field within the element was numerically modelled, both in terms of optimizing its design and with respect to the presence of local defects leading to the formation of partial discharges.

\section{Electric field stress distribution within modules of a gas insulated substation}

The photo in Fig. 1 shows a fragment of a gas insulated module containing a specific example of GIS structure element, which is a spherical connector. The straight modules with a $90^{\circ}$ angular module are the so-called L-shaped connections. 


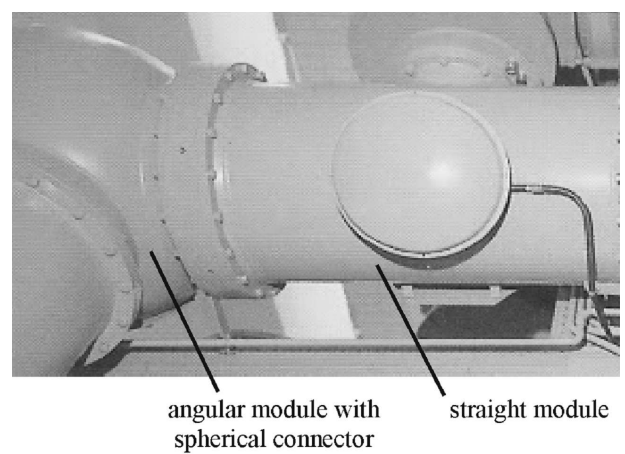

Fig. 1. Spherical connector as a part of 1-phase L-shaped GIS module.

Spherical connectors are located in aluminium or steel tank with $\mathrm{SF}_{6}$ under the pressure from $0.2 \mathrm{MPa}$ up to $0.5 \mathrm{MPa}$ as the main insulating medium. The model of the substation's spherical connector consists of a pair of coaxial cylinders radii $r, R$ (Fig. 2, part I) and concentric spheres radii $r_{k}, R_{k}$ (Fig. 2, part II). The basis of connector designing is the calculation of the maximum value of electric field stress $E_{r}$ at the $\mathrm{HV}$ conductor surface with radius $r$ and electric field stress $E_{r k}$ at the sphere radius $r_{k}$.

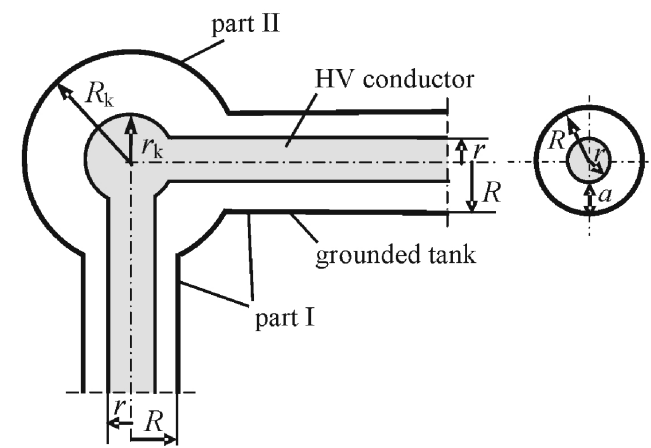

Fig. 2. The model of a spherical connector in L-shaped tank, Part I: coaxial cylinders, $r$ - radius of HV conductor, $R$ - radius of grounded tank, Part II: concentric spheres radii $r_{k}, R_{k}$.

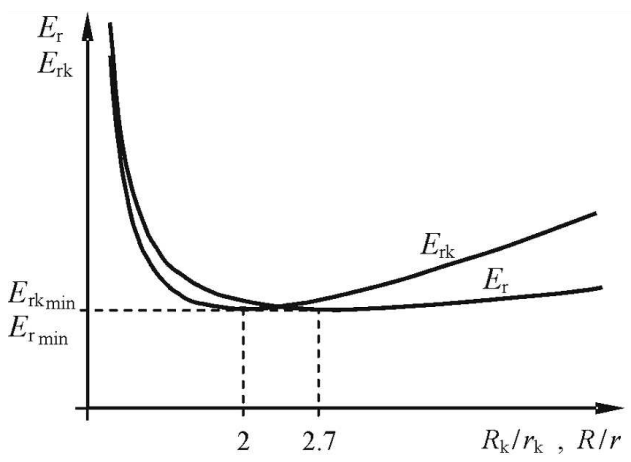

Fig. 3. The electric field values at the surface of inner cylinder $E_{r}=f(R / r)$ and at the surface of inner sphere $E_{r k}=f\left(R_{k} / r_{k}\right)$.
Dimensioning of spherical connector for GIS module working at the rated voltage $U_{n}$ consists in determining the radii $r_{k}, R_{k}$ and $R$ at predetermined permissible value of the electric field $E_{r}$ at the surface of the pipe-type busbar (inner cylinder radius $r$ ) in the straight portion of the connector (part I). The dimensions of concentric spheres, i.e. its radii $R_{k}$ and $r_{k}$ (Fig. 2) should be properly evaluated, so the electric field stress $E_{r}$ at the surface of inner cylinder radius $r$ (conductor) should be equal to the electric field $E_{r k}$ at the surface of the inner sphere radius $r_{k}$ (Fig. 3):

$$
E_{r k \min }=E_{r \min } \text {. }
$$

\section{Part I - model of coaxial cylinders}

The value of the electric field in a coaxial model system at a voltage of $U$, in the area $r<x<R$, according to the Gaussian law is

$$
E_{x}=\frac{Q}{2 \pi \varepsilon l x}
$$

where $Q / l$ - charge per unit length, $\varepsilon-$ dielectric permittivity.

The electric field at the surface of the pipe-type busbar radius $r$ is equal to

$$
E_{r}=\frac{U}{r} \frac{1}{\ln (R / r)}
$$

and its value depends on the ratio of radii $R / r$.

The lowest value of electric field $E_{r}$ min at the rated voltage takes place for $\mathrm{d} E_{r} / \mathrm{d}(R / r)=0$. Then, the ratio of radii $R / r$ is equal to $e$ constant, i.e. the internal diameter $2 R$ of outer cylinder to diameter of the high voltage conductor meets equation $2 R=5.4 r$ (Fig. 3).

\section{Part II - model of concentric spheres}

The electric field in a spherical model system with voltage $U$, in the area $r_{k}<x<R_{k}$, according to the Gaussian law is

$$
E_{x}=\frac{Q}{4 \pi \varepsilon x^{2}} .
$$

The electric field at the surface of the inner sphere radius $r_{k}$ is

$$
E_{r k}=\frac{U}{r_{k}} \cdot \frac{1}{1-\left(r_{k} / R_{k}\right)},
$$

so the value of $E_{r k}$ depends on the ratio of radii $R_{k} / r_{k}$.

The lowest value of the electric field stress at the surface of inner sphere is present when the $\mathrm{d} E_{r k} / \mathrm{d}\left(R_{k} / r_{k}\right)=0$. Then, ratio of radii $R_{k} / r_{k}=2$ (Fig. 3).

The exemplary results of numerically simulated electric field distribution for L-shaped tank model obtained by the FEM method with the ANSYS software are shown in Figs. 4 and 5.

The result of computer simulation for optimal construction of L-shaped tank geometry (Fig. 4) is the electric field image (Fig. 5) with uniformly distributed electric field stress on the surface of the high voltage conductor and on the spherical connector. Precise numerical modelling of the GIS module geometry allows for optimizing the electric field distribution on the surfaces of the individual components at the design stage. 


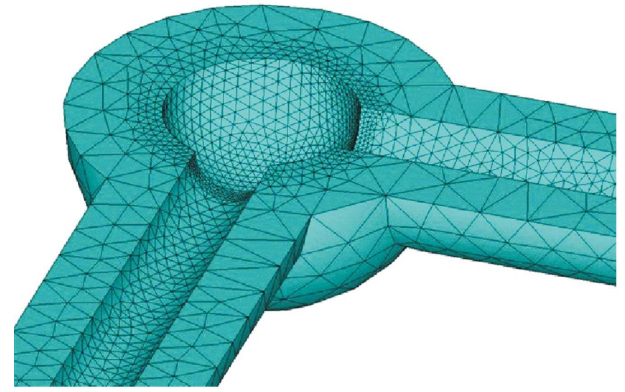

Fig. 4. Numerical modelling of spherical connector geometry in L-shape tank.

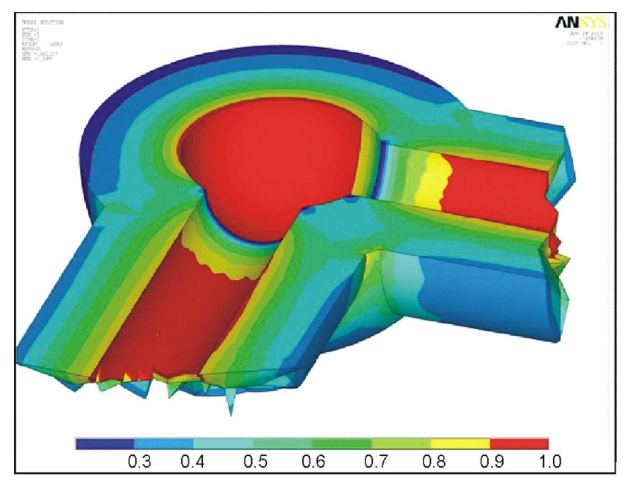

Fig. 5. The image of the electric field stress in optimal model of spherical connector.

Software for modelling of electric fields is thus a powerful tool that supports the processes of designing and preliminary evaluation of the correctness of the project. In every case the final verification lies in real $\mathrm{HV}$ tests performed on prototype devices in laboratory conditions.

\section{Modelling of electric field distribution in the vicinity of local sources of partial discharges}

The maximum values of the electric field in spherical connectors ensure their normal operation at rated voltage gas insulated substations. This state can be affected by defects in the gas insulated system in its modules which cause a local increase of the electric field. Defects in a substation module are the sources of partial discharges in this construction. One can distinguish the 'stable' defects and the 'dynamic' ones, which are free conductive particles moving in the gas space or on the surface of conductive elements and insulators (Fig. 6).

Basic kinds of defects are:

1. micro-needle on the high voltage conductor $\left(1^{\prime}\right)$ or on the grounded tank $\left(1^{\prime \prime}\right)$,

2. free-moving particles in gaseous space with floating potential (2),

3. conductive particles/contaminations on the insulator surface (3),

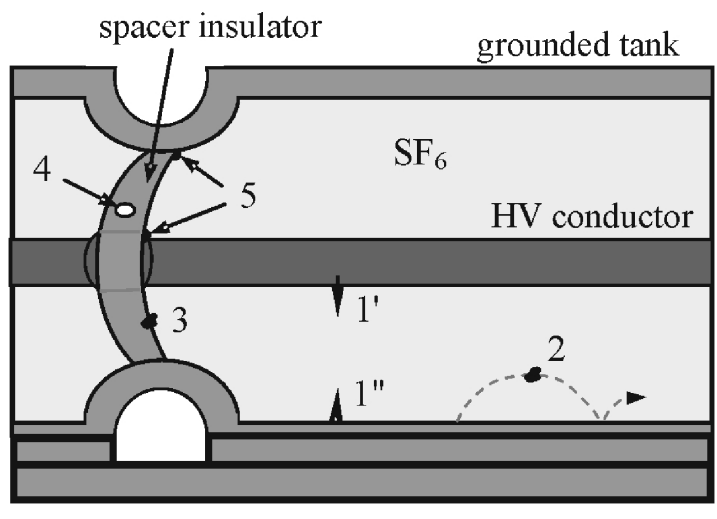

Fig. 6. Part of the single-phase substation module and possible location of different defects.

4. gaseous inclusion in solid insulation of the spacer insulating material (4),

5. particles in the "triple point" (5).

Typically, above-mentioned defects have an effect on the electric field distribution in the substation module. Moreover, the local electric field stress can be higher than the partial discharge inception one.

Each defect, which can potentially generate discharges, both during the post-installation tests or during normal working of substation, can be classified as critical because it results in decrease of withstand voltage below the coordination voltage value [19]. This value equals to the lowest substation withstand voltage value in the determined time of its exploitation.

Particular hazard in $\mathrm{SF}_{6}$ systems stems from the overvoltages which can incept discharges and as a result change the physical state of the insulating gas.

The main aims of the diagnostic system of high voltage insulation based on partial discharges are:

- detection of partial discharges in the presence of noises and disturbances,

- identification and localisation of partial discharges sources linked to GIS defects,

- on-line monitoring and assessment of partial discharges in working GIS.

Next follows the analysis of impact of defect type 1 (Fig. 6) on the distribution of the electric field at the surface of the conductor and the possibility of initiating PD. The following parameters are determined in Fig. 7: $E(x)$ - electric field distribution in $\mathrm{SF}_{6}$ gas between the conductor and grounded tank, $E_{d}(x)$ - electric field distribution in the vicinity of defect on the conductor surface.

Local increase of electric field in the vicinity of microneedle on the high voltage conductor (Fig. 7) is described by the coefficient $\beta_{r}=E_{r d} / E_{r}$ (where $E_{r d}$ - the highest value of electric field at micro-needle defect; $E_{r}-$ maximal value of electric field at the conductor surface). 
The value of $\beta_{r}$ depends on the radius of defect curvature $r_{d}$, while its shape has no essential meaning.

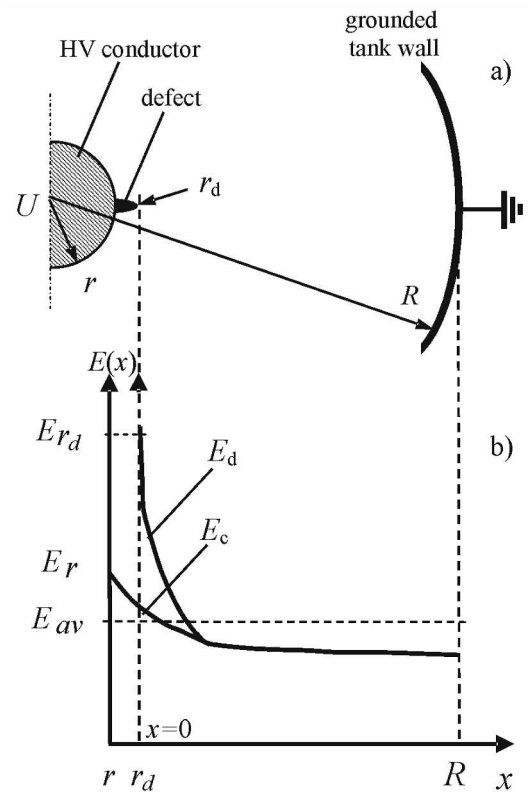

Fig. 7. Model of coaxial cylinders: (a) defect with radius $r_{d}$ on high voltage conductor, (b) electric field distribution in the vicinity of conductor $E(x)$ and at the tip of the micro-needle defect $E_{d}(x)$.

A model of micro-needle defect has been adopted as hyperboloidal electrode in above-mentioned case [20]. Here the electric field stress at a distance $x$ from the needle tip equals to

$$
E(x)=\frac{2 U}{\left(2 x+r_{d}-x^{2} / a\right) \ln \left(4 a / r_{d}\right)} .
$$

for $0 \leq x \leq\left(R-r_{d}\right)$, where $U$ - voltage between the needle and grounded plane electrode, $a$ - distance $(a=R-r)$.

Electric field stress at the point $x=0$ is expressed by equation

$$
E_{r d}=\frac{2 U}{r_{d} \ln \left(4 a / r_{d}\right)} .
$$

Relations $E_{d}(x)$ evaluated for the assumed reference voltage value $U=1 \mathrm{kV}$ and for defect radius $r_{d}$ in the range from $50 \mu \mathrm{m}$ to $500 \mu \mathrm{m}$ (Fig. 7) show to the existence of a region at a distance to about $10^{-1} \mathrm{~cm}$, i.e. zone of ionisation and space charge near the needle electrode (Fig. 8). The region at a distance greater than about $1 \mathrm{~cm}$ does not influence the distribution of electric field in the vicinity of the needle of different curvature.

The electric field distribution in the vicinity of the needle-type defect on the conductor surface has been modelled on the basis of the following assumptions:

- height of defect $h$ equals to 1,3 , or $5 \mathrm{~mm}$,

- curvature radius $r_{d}$ equals to 50,100 , or $200 \mu \mathrm{m}$.

In a typical substation with the rated voltage of $340 \mathrm{kV}$, the radius of high voltage conductor is $r=55 \mathrm{~mm}$ and

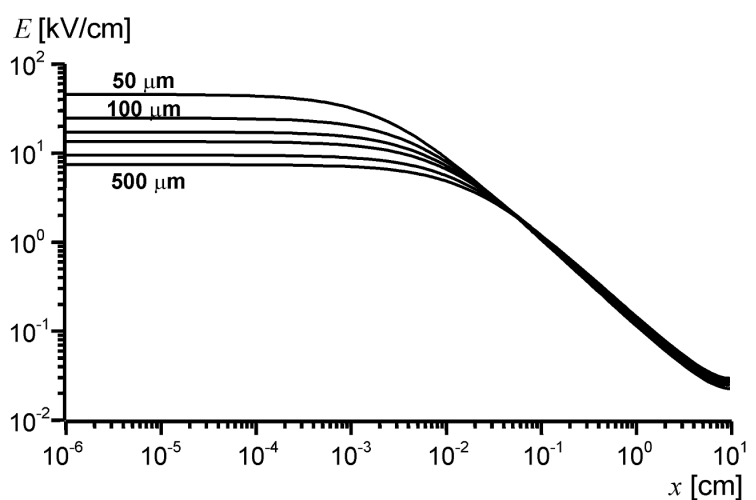

Fig. 8. The electric field values in the distance of $x$ from needle with different curvatures $r_{d}$ from $50 \mu \mathrm{m}$ to $500 \mu \mathrm{m}$ (calculated for $U=1 \mathrm{kV}$ ).

the radius of outer electrode (metallic enclosure of GIS module) is $R=152 \mathrm{~mm}$. Exemplary result of numerical simulation of the electric field spatial distribution in the vicinity of the needle defect located on high voltage conductor is presented in Fig. 9.

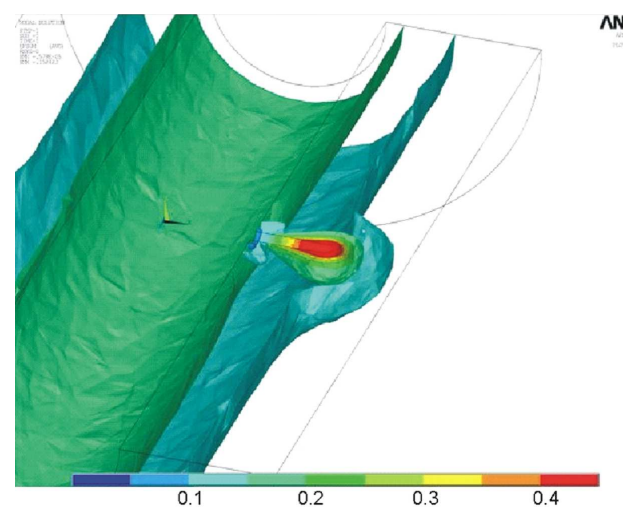

Fig. 9. Example of electric field stress distribution in the vicinity of the needle defect on the HV conductor in the coaxial cylinders.

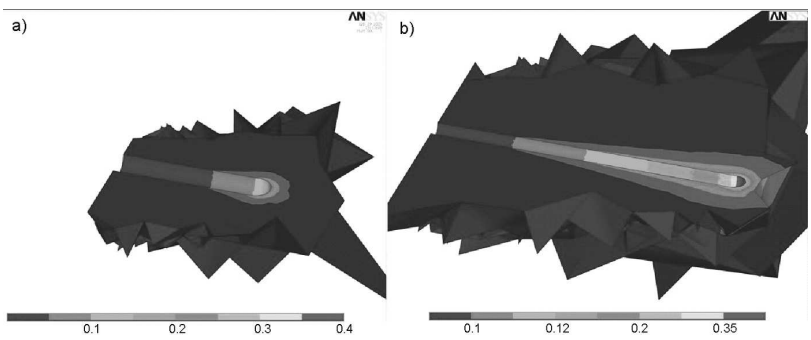

Fig. 10. Images of electric field in the vicinity of defects on the conductor $\left(r_{d}=100 \mu \mathrm{m}\right)$ for different height of defects: a) $h=1 \mathrm{~mm}$, b) $h=5 \mathrm{~mm}$.

Patterns of electric field stress presented in Fig. 10 illustrate two selected cases when the electric field stress $E_{r d}$ does not excess the critical value, at which partial discharges can occur (defect: height $h=1 \mathrm{~mm}, r_{d}=$ $100 \mu \mathrm{m}$ ) (Fig. 10a), and when it goes beyond this value 
because of the higher needle-type defect $(h=5 \mathrm{~mm}$, $\left.r_{d}=100 \mu \mathrm{m}\right)$ (Fig. 10b).

\section{Mechanism of partial discharges in $\mathbf{S F}_{6}$}

Analysing the phenomena of partial discharges initiation and development in GIS one should take into account the construction of the object and other physical conditions, which are

- defined electrode configuration,

- defect kind as the source of partial discharges, with different conditions of discharges initiation,

- possibilities of avalanche and streamer discharges development,

- pulse form of these phenomena.

The discharge pulse consists of streamers propagating into gas from the tip of the micro-defect to a limited distance in which the local electric field is equal or higher than a critical value $E_{c r}$. This value in $\mathrm{SF}_{6}$ is related to the pressure of gas [21]. When $E \geq E_{c r}$ criterion is fulfilled, the mechanism of PD initiation depends on the polarity of the micro-needle:

- at the positive polarity - electrons are produced by the field detachment from negative ions,

- at negative polarity - the dominant phenomenon is the field emission from the metallic surface of the defects.

Conducting particles located on insulator surface or in the "triple point" enable the evolution of surface discharges. On the other hand, inner inclusions in insulators are the cause of inner erosion and evolution of treeing discharges.

\subsection{Initiation of partial discharges from micro-needles}

In the case of micro-needles on the surface of a conductor (Figs. 6 and 7 ) the conditions for discharges initiation stem from the field emission and tunnel effect at the micro-needle. The probability of field emission on the conductor/gas interface depends on the value of electric field stress $E_{d}$, at the surface of micro-needle (Fig. 11). The increase of electric field stress at the needle in relation to the average electric field stress $(E=U / a)$ is expressed by the coefficient $\beta=E_{d} / E$. The current efficiency of the field emission depends on the following factors:

- local electric field stress at the micro-needle $E_{d}$,

- electric field stress in the insulating system $E$,

- effective work of electron emission from metal expressed by the Fowler-Nordheim formula [22]:

$$
N_{\mathrm{eFN}}=\pi r^{2} A_{1} \frac{\beta^{2} E^{2}}{\Phi_{\mathrm{ef}}} \exp \left[-\frac{A_{2} \Phi_{\mathrm{ef}}^{3 / 2}}{\beta E}\right],
$$

where $\Phi_{\text {ef }}$ - effective work of electron emission from cathode $[\mathrm{eV}], A_{1}, A_{2}-$ constants: $A_{1}=1.54 \times$ $10^{-6} \mathrm{eV} \mathrm{A} \mathrm{V}^{-2}, A_{2}=6.79 \times 10^{9}(\mathrm{eV})^{-3 / 2} \mathrm{~V} \mathrm{~m}^{-1}$.

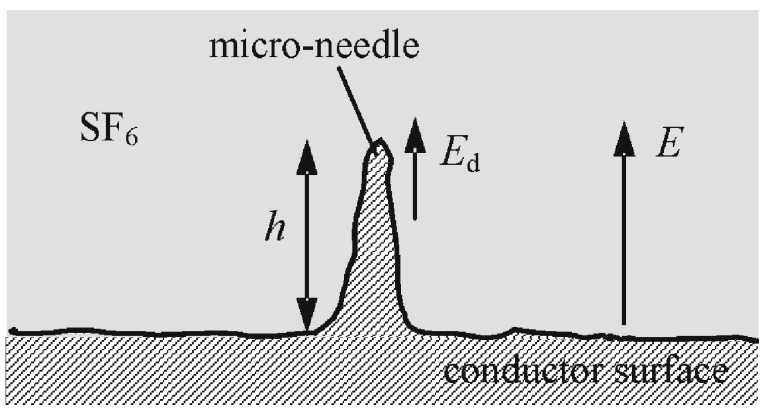

Fig. 11. Model of a micro-needle on the conductor surface $(\mathrm{CS})$.

The tunnel effect is most intense at the top of the micro-needle, but the height of the micro-needle should be so determined as to make this effect appear. It is connected with the shape and width of the potential barrier at the metal surface as they are needed to evolve this effect. The value of electron emission work $\Phi_{\text {ef }}$ depends on the stage of micro-needle surface, particularly on the presence of adsorbed atoms of other elements which can lower it or rise and also on the kind of interfaces, e.g. metal/gas, metal/solid dielectric. Abovementioned problems are of particular meaning for the initiation of discharges in vacuum insulating systems. Thus the condition of discharges initiation due to field emission results from the relation $N_{\mathrm{eFN}}=f(\beta E)$. Formula (7) indicates a strong dependence of field emission on the product $\beta E$ so that for $\beta E<(\beta E) *$ emission practically does not exist and for $\beta E>(\beta E) *$ it is intensive, while $(\beta E) *$ is the threshold value between the initial and intensive emission. To evaluate the threshold value $(\beta E) *$ one should account for the emission of only one electron during one half-period $T$ of AC voltage, i.e. $N_{\mathrm{eFN}}=2 e / T$ ( $e$ is the elementary charge). Then

$$
(\beta E) *=\frac{A_{2} \Phi_{\mathrm{ef}}^{3 / 2}}{\ln \left(A_{1} \pi r^{2} \frac{T}{2 e} \frac{[(\beta E) *]^{2}}{\Phi}\right)} .
$$

Basing on the assumption that the work functions $\Phi_{\text {ef }}$ of electrons from metal stays in the range $3 \div 5 \mathrm{eV}$ for micro-needles in vacuum, and $0.9 \div 1.5 \mathrm{eV}$ for microneedles in solid dielectric, time $T / 2=10 \mathrm{~ms}$, and the top surface of the micro-needle is $0.1 \mathrm{~mm}^{2}$ to $1 \mathrm{~mm}^{2}$, two relationships $(\beta E) *=f\left(\Phi_{\mathrm{ef}}\right)$ and $E_{d}=f\left(\Phi_{\mathrm{ef}}\right)$ have been worked out (Fig. 12) [18]. Threshold values of $(\beta E) *$ range from $6 \times 10^{8} \mathrm{~V} \mathrm{~m}^{-1}$ to $15 \times 10^{8} \mathrm{~V} \mathrm{~m}^{-1}$ - for microneedle in gas, and from $1 \times 10^{8} \mathrm{~V} \mathrm{~m}^{-1}$ to $3 \times 10^{8} \mathrm{~V} \mathrm{~m}^{-1}$ - for micro-needle in solid dielectric, respectively. 


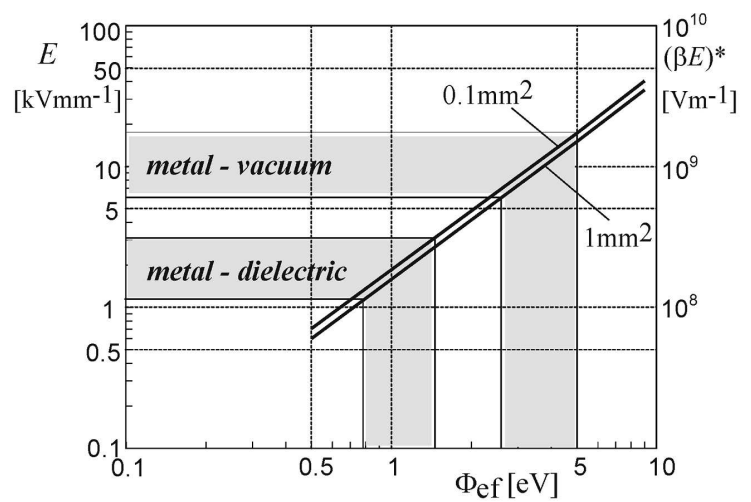

Fig. 12. Dependence of threshold value $(\beta E) *$ and the electric field stress $E$ on the work of electron emission from metal for surfaces $0.1 \mathrm{~mm}^{2}$ and $1 \mathrm{~mm}^{2}$.

If the coefficient of micro-geometry is equal to, e.g. $\beta=100$, then the values of electric field stress range from $6 \times 10^{6} \mathrm{~V} \mathrm{~m}^{-1}$ to $15 \times 10^{6} \mathrm{~V} \mathrm{~m}^{-1}$ (i.e., $6 \mathrm{kV} / \mathrm{mm}$ to $15 \mathrm{kV} / \mathrm{mm}$ ), and from $1 \times 10^{6} \mathrm{~V} \mathrm{~m}^{-1}$ to $3 \times 10^{6} \mathrm{~V} \mathrm{~m}^{-1}$ (i.e., $1 \mathrm{kV} / \mathrm{mm}$ to $3 \mathrm{kV} / \mathrm{mm}$ ). These are values of working electric field stress in insulating systems.

\subsection{Discharges development}

Space development of electron avalanches in gas depends on impact ionisation processes characterised by coefficient $\alpha$, and in the case of electronegative gases as $\mathrm{SF}_{6}$, also by electron attachment coefficient $\eta$, which influences the number of electrons in an avalanche. The capture of electrons in $\mathrm{SF}_{6}$, when $\eta \neq 0$ results in the fact that the avalanche development is possible only for $\alpha>\eta$.

Further development of discharge in the form of streamers in the limited space in gas above the top of micro-needle is connected with the self-propagation of electron avalanches as a result of field increase in the region of space charge ionisation. Local field stress is then greater than the critical value $E_{c r}$. On the other hand, this value depends on gas pressure. The development of electron avalanches is possible when

$$
(E / p)>(E / p)_{c r} .
$$

In electrodes with homogeneous or semi-homogeneous field in sulphur hexafluoride the value $(E / p)_{c r}$ in the temperature $300 \mathrm{~K}$ equals to $89 \mathrm{~V} \mathrm{~m}^{-1} \mathrm{~Pa}^{-1}$ [21]. The possible deformation of local electric field distribution and the overvoltages in exploitation in view, the electric field stress on the HV conductor surface can be assumed not to exceed $8.9 \mathrm{~V} \mathrm{~m}^{-1} \mathrm{~Pa}^{-1}$ [23]. If the gas pressure in the substation is $0.5 \mathrm{MPa}$, electric field stress at the $\mathrm{HV}$ conductor equals to $4.5 \mathrm{kV} / \mathrm{mm}$. The direction and area of the streamer discharge propagation depend on the kind of defect in the substation.

In the case of micro-needle defects located on the conducting elements the three-dimensional space of discharges in gas can be approximately modelled as the conducting sphere which cumulates the discharge charge. When the defects are conducting particles adherent to the insulator surface, then it is approximately a twodimensional area on the insulator surface which cumulates the charge. Different kinds of discharge are produced as the effect of discharges initiated by above mentioned defects: in the first case they could be similar to corona discharge, in the second - similar to the surface ones.

\section{Final remarks}

Partial discharges in gas insulated substations may exist because of different imperfections (micro-needles, free particles, contaminations) in the substation modules. Discharges are generated when the critical value of electric field is achieved at any point located inside the gaseous HV insulating system. Computer simulation of micro-needle model defects by means of finite element method is a tool for determining conditions for partial discharges initiation when sufficiently high electric field is reached. It was shown during modelling that such a metallic defect on a high voltage conductor, working at defined voltage, could be a local source of partial discharges when it has specific geometrical dimensions.

\section{References}

[1] U. Schichler, W. Koltunowicz, F. Endo, K. Feser, A. Giboulet, A. Girodet, H. Hama, B. Hampton, H.G. Kranz, J. Lopez-Roldan, L. Lundgaard, S. Meijer, C. Neumann, S. Okabe, J. Pearson, R. Pietsch, U. Riechert, S. Tenbohlen, IEEE Trans. Dielectr. Electr. Insul. 20, 2165 (2013).

[2] CIGRE WG D1.03-TF 09, CIGRE Technical Brochure 525, 1 (2013).

[3] W. Koltunowicz, Diagnostic Insulation Testing of High Voltage Gas Insulated Substation, Oficyna Wyd. Politechniki Warszawskiej, Warszawa 2003 (in Polish).

[4] K. Dreisbusch, H.-G. Kranz, A. Schnettler, IEEE Trans. Dielectr. Electr. Insul. 15, 1707 (2008).

[5] M. Visintin, L. Niemayer, G. Rabach, Proc. IEEE ISEI, Pittsburg, USA 1994, p. 277.

[6] J.S. Pearson, O. Farish, B.F. Hampton, M.D. Judd, D. Templeton, B.W. Pryor, I.M. Welch, IEEE Trans. Dielectr. Electr. Insul. 2, 893 (1995).

[7] M.D. Judd, O. Farish, B.F. Hampton, IEEE Trans. Dielectr. Electr. Insul. 3, 213 (1996).

[8] H. Okubo, T. Kato, N. Hayakawa, M. Hikita, IEEE Trans. Power Delivery 13, 440 (1998).

[9] I.A. Metwally, J. Electric Power Syst. Res. 69, 25 (2004).

[10] M. Muhr, T. Strehl, E. Gulski, K. Feser, E. Gockenbach, W. Hauschild, E. Lemke, Proc. CIGRE, Paper D1-102, Paris 2006.

[11] S. Okabe, S. Kaneko, M. Yoshimura, H. Muto, C. Nishida, M. Kamei, IEEE Trans. Dielectr. Electr. Insul. 14, 702 (2007).

[12] M. Yoshida, H. Kojima, N. Hayakawa, F. Endo, H. Okubo, IEEE Trans. Dielectr. Electr. Insul. 18, 425 (2011). 
[13] B. Florkowska, M. Florkowski, R. Włodek, P. Zydroń, Mechanisms, Measurements and Analysis of Partial Discharges in Diagnostics of High Voltage Insulating Systems, IPPT PAN Press, Warszawa 2001 (in Polish).

[14] R. Kurte, C. Beyer, H.M. Heise, D. Klockow, Anal. Bioanal. Chem. 373, 639 (2002).

[15] W. Ding, K. Ochi, J. Suehiro, K. Imasaka, R. Hayashi, M. Hara, IEEE Trans. Dielectr. Electr. Insul. 14, 718 (2007).

[16] Z. Gacek, Shaping of High Voltage Insulation Systems Applied in Electric Power Industry, Wyd. Politechniki Śląskiej, Gliwice, 2002 (in Polish).

[17] B. Florkowska, P. Zydron, Przeglad Elektrotechn. Konf. 3, 94 (2005) (in Polish).

[18] B. Florkowska, Partial Discharges in High Voltage Insulating Systems - Analysis of Mechanisms, Forms and Patterns, IPPT PAN Press, Warszawa, 1997 (in Polish).
[19] IEC 60071-1:2006 Insulation coordination. Part 1: Definitions, principles and rules, 2006.

[20] B. Florkowska, R. Włodek, IEEE Trans. Electr. Insul. 28, 932 (1993).

[21] E. Kuffel, W.S. Zaengl, J. Kuffel, High Voltage Engineering - Fundamentals, Butterworth-Heinemann, Oxford, 2000.

[22] A. Murray, P. Mark, Current Injection in Solids, Academy Press, New York 1970.

[23] J. Maksymiuk, Z. Pochanke, Calculations and Diagnostic Testing of Distribution Equipment, WNT, Warszawa 2001 (in Polish). 